\title{
Editorial: Skull, bone growth, skull basis anatomy, foramina, canals and crossing structures: so often considered as one of the most difficult part in medical education!
}

\author{
Fabrice Duparc ${ }^{1} \cdot$ Bruno Grignon $^{2} \cdot$ Figen Govsa $^{3}$
}

Published online: 27 May 2020

(c) Springer-Verlag France SAS, part of Springer Nature 2020

When it comes to learning the bones of the skull, students are usually facing difficulties, and teachers are continuously trying to facilitate this section of anatomical education. This is the main reason behind our proposal which includes in the first part of this issue some articles on skull bones, growth of some of them, foramina of the skull basis and their contents, and to compile in the second part some articles devoted to the use of new tools for the three dimensional (3D) depiction of anatomical features.

Teaching skull basis anatomy means concurrently teaching fibrous structures as dura-mater and cartilage, and nerves and vessels coursing from the cranial fossae to the face and neck. It is important to understand the bones growth through the development of primary centers of ossification and the growth pattern of neurocranial bones can be depicted by finite elements analysis. The correlation of the osseous growth and the nervous and vascular development determine the anatomical conditions in adults, and the article on the growth dynamic of external aperture of the internal carotid may reflect the progress of the skull basis providing safety landmarks for surgery.

The zones of communications between the cranial cavities and the face and neck represent some examples of this correlated growth. The cavernous sinus is an example of the complex joint learning of bones, dura, veins and nerves. The greater wing of the sphenoid is a piece of bone involved in several foramina. Among them, the foramen ovale has been studied by contrast-MRI with special attention to the emissary veins venous channels that run close to the nerve- $\mathrm{V}_{3}$. The foramen venosum, so well depicted by Vesalius, is a

Fabrice Duparc

fabrice.duparc@univ-rouen.fr

1 Faculty of Medicine, Rouen-Normandy University, Mont-Saint-Aignan, France

2 Department of Anatomy, University of Lorraine, Vandoeuvre-Les-Nancy, France

3 Faculty of Medicine, Ege University, Bornova, Izmir, Turkey very frequent anatomical variation. The anatomical research of safety conditions in clinical practice is a constant goal in clinical anatomy: the absence of direct relative positional relationship between the external auditory meatus and sigmoid sinus is an example of applied anatomy. The craniometry of the craniovertebral junction leads to standard parameters that can be correlated to age and gender, with interest in surgical practice and in imaging, but also in forensics.

After passing through the cranial cavity and skull basis, the nerves continue to travel to their targets through bony canals of the face. The nasopalatine canal is of interest in endoscopic procedures and anatomical landmarks are useful and warrant of safe techniques. The zygomatic bone is an important part of the periorbital region surgery, but at risk of zygomaticofacial nerve or blood vessel injury justifying the anatomical assessment of criterions for secured procedure.

The knowledge of anatomical variations is not only a part of the anatomical culture, but it is essential for the specialists of different anatomical regions and for interpretation in medical imaging.

As these sections of anatomy are often considered difficult by the students, we have completed this issue with a few articles devoted to medical and anatomy education.

3-D-printing seems like an interesting tool for facilitating the learning process of the anatomical features, and moreover 3D-stereoscopic-video contributed to a solid understanding of the anatomy. Particularly 3D-vizualisation techniques, which are in constant progress and need to be evaluated as they represent very useful tools for completing the traditional teaching of anatomy.

The transformation of the human dissection leads to actual new aspects of ethics in anatomical practice, research and education.

We wish you a very stimulating reading!

Publisher's Note Springer Nature remains neutral with regard to jurisdictional claims in published maps and institutional affiliations. 